Novel high performance small-scale thermoelectric power generation employing regenerative combustion systems

This article has been downloaded from IOPscience. Please scroll down to see the full text article.

2002 J. Phys. D: Appl. Phys. 35 L61

(http://iopscience.iop.org/0022-3727/35/13/102)

View the table of contents for this issue, or go to the journal homepage for more

Download details:

IP Address: 131.251.133.27

The article was downloaded on 02/04/2012 at 10:48

Please note that terms and conditions apply. 


\title{
Novel high performance small-scale thermoelectric power generation employing regenerative combustion systems
}

\author{
F J Weinberg ${ }^{1}$, D M Rowe ${ }^{2}$ and G Min ${ }^{2}$ \\ ${ }^{1}$ Imperial College, London SW7 2BY, UK \\ ${ }^{2}$ NEDO Laboratory, School of Engineering, Cardiff University, Cardiff CF24 3TF, UK
}

Received 10 December 2001

Published 18 June 2002

Online at stacks.iop.org/JPhysD/35/L61

\begin{abstract}
Hydrocarbon fuels have specific energy contents some two orders of magnitude greater than any electrical storage device. They therefore proffer an ideal source in the universal quest for compact, lightweight, long-lasting alternatives for batteries to power the ever-proliferating electronic devices. The motivation lies in the need to power, for example, equipment for infantry troops, for weather stations and buoys in polar regions which need to signal their readings intermittently to passing satellites, unattended over long periods, and many others. Fuel cells, converters based on miniaturized gas turbines, and other systems under intensive study, give rise to diverse practical difficulties. Thermoelectric devices are robust, durable and have no moving parts, but tend to be exceedingly inefficient. We propose regenerative combustion systems which mitigate this impediment and are likely to make high performance small-scale thermoelectric power generation applicable in practice. The efficiency of a thermoelectric generating system using preheat when operated between ambient and $1200 \mathrm{~K}$ is calculated to exceed the efficiency of the best present day thermoelectric conversion system by more than $20 \%$.
\end{abstract}

\section{Introduction}

Over thirty years ago [1] it was shown that combustion systems in which reactants, or the air alone, are preheated using heat 'borrowed' from downstream of the flame, so as to increase the flame temperature substantially, can burn remarkably fuel-lean mixtures and offer significant advantages as regards fuel conservation, efficiency and combustion intensity [2]. Subsequent publications [3,4] highlighted their potential for enhancing the efficiency of energy extraction. In view of the inefficiency of thermoelectric devices, it should be possible to recycle a large proportion of the heat rejected at their cold junctions. This suggests associating them with the kind of counterflow heat exchanger that underlies heat recirculating combustion systems. We report here the results of a theoretical study to establish limits to conversion efficiencies for such systems.

\section{Design considerations and results}

The design is illustrated in figure 1 . The temperatures, $T$, at strategic points are denoted by suffixes as follows: $0=$ ambient, $\mathrm{u}=$ unburnt (just upstream of flame), $\mathrm{b}=$ burnt (just downstream of flame), $\mathrm{f}=$ final (at heat exchanger exit). The mixture consists mostly of air $(>98.4 \%)$ and even $T_{\mathrm{b}}$ is too low for dissociation. For a particular specific heat capacity, $c$, and mass flow rate, $M$, both assumed constant, we can arrange the dimensions and geometry so as to give 


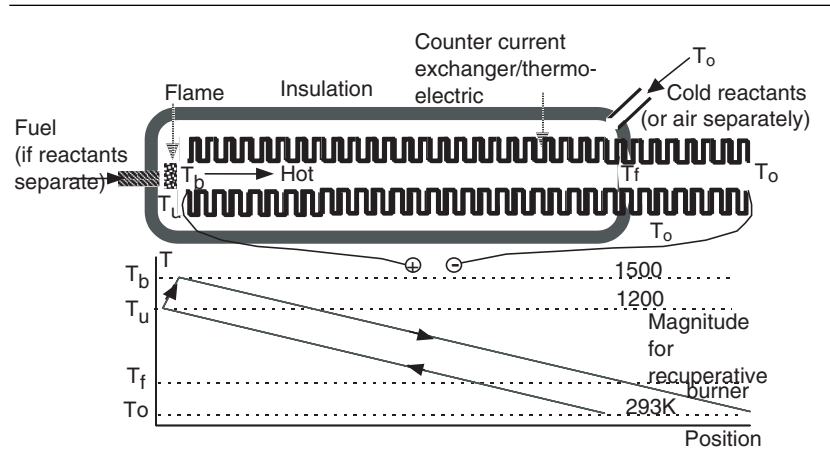

Figure 1. Schematic of combustion/converter system.

the specified temperature intervals. The chemical heat release rate is $M f Q$ ( $Q=$ heat of combustion/mass of stoichiometric mixture, $f=$ fraction of stoichiometric fuel concentration) and heat losses from the heavily insulated system are neglected.

The heat exchanger/thermoelectric converter consists of a coaxial assembly of a large number of flat annular 'washers' consisting of alternating n-type and p-type thermoelectric materials connected in series. They are joined alternately at their inner and outer peripheries, in the manner of compressed concertina bellows. Except at these hot and cold junctions, they are separated by insulating material, prior to being compressed axially. The cold junctions act as the inlet limb of the heat exchanger, except for the protrusion where they are maintained at ambient temperature. This external stage is necessary because the exit temperature from the heat recirculating stage, $T_{\mathrm{f}}$, is determined by the heat that must flow out, because of the inefficiency of the system, in order to maintain the steady state. However, there is no reason why we cannot discard the unconverted heat through the external section of the converter, so long its cold side is at $T_{\mathrm{o}}$ and not part of the heat recycle. In practice, this only requires the converter protrusion beyond $T_{\mathrm{f}}$ to be long enough to cool the product gases to near ambient temperature, $T_{0}$. The thermoelectric materials can be varied along the concertina-like structure to obtain optimum efficiency for different temperature ranges.

The absolute maximum efficiencies permitted by the second law, used as a guideline for comparison with less efficient irreversible converters, can be calculated by supposing each thermoelectric element to be replaced by an elementary Carnot cycle of efficiency:

$$
\eta=1-\frac{T_{\text {cold }}}{T_{\text {hot }}} .
$$

The integration to obtain overall system efficiencies, $\eta_{\mathrm{g}}$, over a range of temperatures has been carried out previously [4, 5], both for the case of heat rejection to the incoming reactants, as in the first stage of figure 1, and for that of heat rejection to the ambient cold surroundings, $T_{\mathrm{o}}$, as in the external stage. For the temperatures specified in figure 1 , the results are, respectively,

$$
\eta_{\mathrm{g}}=1-\frac{T_{\mathrm{u}}}{T_{\mathrm{b}}}=1-\frac{T_{\mathrm{o}}}{T_{\mathrm{f}}},
$$

which also specifies

$$
T_{\mathrm{f}}=\frac{T_{\mathrm{o}} T_{\mathrm{b}}}{T_{\mathrm{u}}}
$$

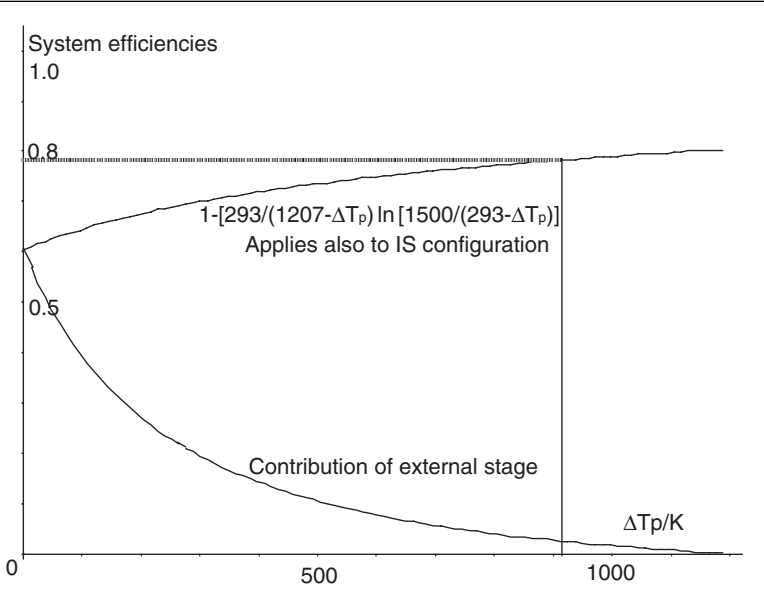

Figure 2. 'Carnot' system efficiencies vs preheat, $\Delta T_{\mathrm{p}}\left(=T_{\mathrm{u}}-T_{\mathrm{o}}\right)$.

and

$$
\eta_{\mathrm{g}}=1-\frac{T_{\mathrm{o}}}{T_{\mathrm{f}}-T_{\mathrm{o}}} \ln \left(\frac{T_{\mathrm{f}}}{T_{\mathrm{o}}}\right) .
$$

Good heat re-circulating burners typically achieve [2,5] $T_{\mathrm{u}}=1200 \mathrm{~K}$ so that a combustion heat release corresponding to a temperature rise of only $300 \mathrm{~K}$ is necessary to reach the $1500 \mathrm{~K}$ level required to sustain a stable flame. For $T_{\mathrm{o}}=293 \mathrm{~K}$, the corresponding $T_{\mathrm{f}}=293 \times 1500 / 1200=$ $366.25 \mathrm{~K}$. The overall system efficiencies of the two stages are therefore 0.2 and 0.1074 for the internal and external stages, respectively. Multiplying each of them by their heat input rate and dividing by that released by combustion gives an overall system efficiency of $(0.2 \times 1133.75+0.1074 \times 73.25) / 300=$ 0.782 .

Note that this is identical to the efficiency of an idealized system (hereafter IS) which would intuitively be expected to yield an absolute efficiency maximum: a hypothetically perfect heat exchanger that operates on an infinitesimal temperature difference, surmounted by a thermoelectric converter which uses all the heat of combustion at the maximum temperature and rejects heat to the ambient cold surroundings. This IS configuration has a higher $\eta_{\mathrm{g}}$, due to its lower sink temperature, but works on a smaller heat flow. Its system efficiency is $1-(293 /(1500-1200) \ln (1500 / 1200))=0.782$. It can be shown that this identity applies irrespective of the amount of preheating-unsurprisingly perhaps, the maximum conversion permitted by the second law proves to be independent of configuration.

The effect of varying preheat for these second law optima is illustrated in figure 2 . The maximum system efficiency without preheat (requiring an approximately fivefold greater fuel concentration to burn) at $1-(293 /(1500-$ 293) $\ln (1500 / 293))=0.604$ is seen to be about three-fourth of the above.

Next, we assess our assembly of semiconductor thermoelectric junctions in the same way. The maximum efficiency of a thermoelectric device for hot and cold temperatures $T_{\mathrm{h}}$ and $T_{\mathrm{c}}$ is conventionally expressed [6] as

$$
\eta=\frac{T_{\mathrm{h}}-T_{\mathrm{c}}}{T_{\mathrm{h}}} \frac{\sqrt{1+Z T_{\mathrm{a}}}-1}{\sqrt{1+Z T_{\mathrm{a}}}+T_{\mathrm{c}} / T_{\mathrm{h}}},
$$

where $T_{\mathrm{a}}=\left(T_{\mathrm{h}}+T_{\mathrm{c}}\right) / 2$, and $Z\left(=\alpha^{2} \sigma / \lambda\right)$, the 'thermoelectric figure-of-merit', is a measure of the effectiveness of the 


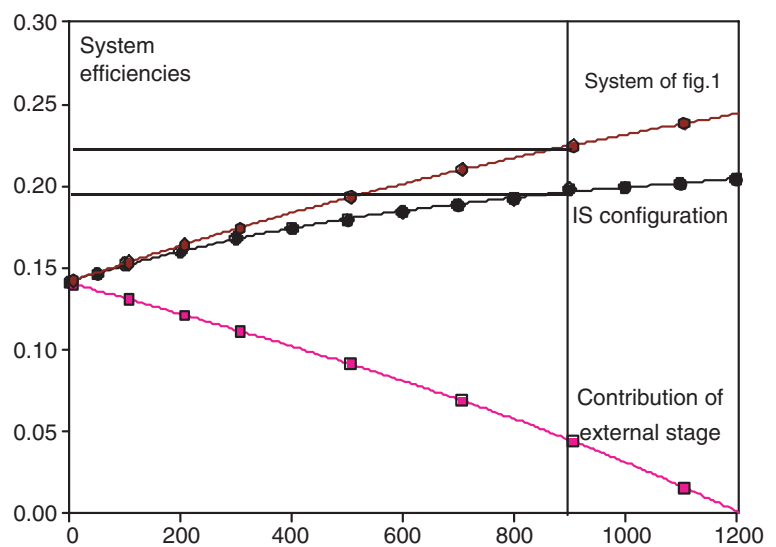

Figure 3. Thermoelectric efficiencies vs preheat, $\Delta T_{\mathrm{p}}\left(=T_{\mathrm{u}}-T_{\mathrm{o}}\right)$.

thermoelectric materials for energy conversion $(\alpha=$ Seebeck coefficient, $\sigma=$ electrical conductivity and $\lambda=$ thermal conductivity). Since our objective is to calculate maximum attainable efficiencies and since we have the freedom to vary the thermoelectric materials (including segmented configurations) along the train so as to optimize efficiency for different temperature ranges, we put $Z T=1$ as a typical maximum value for known thermoelectric materials. Thus

$$
\eta=\frac{T_{\mathrm{h}}-T_{\mathrm{c}}}{3.414 T_{\mathrm{h}}+2.414 T_{\mathrm{c}}} .
$$

We use the same temperatures as in the 'Carnot case', except for $T_{\mathrm{f}}$ which will clearly be higher, since the lesser efficiency will require more heat to be discarded at the exit from the first stage. In this instance it is easier to solve the relationships numerically rather than analytically. The relationship between $T_{\mathrm{f}}$ and $T_{\mathrm{o}}$ turns out to be almost linear in the range of interest, being well represented by

$$
T_{\mathrm{c}}=0.945 T_{\mathrm{h}}-221,
$$

so that, for $T_{\mathrm{o}}=293 \mathrm{~K}, T_{\mathrm{f}}=544 \mathrm{~K}$. The anticipated larger $T_{\mathrm{f}}$ significantly enhances the contribution of the external stage, resulting in an overall system efficiency,

$$
\eta_{\mathrm{g}}=\frac{13.8+54.2}{300}=0.226
$$

Unlike in the case of Carnot cycles, this is substantially more than the IS configuration efficiency, at 0.197 for our $T_{\mathrm{u}}$. Figure 3 shows the variation with preheat. Using approximately one-fourth of the fuel concentration, the increase in the system efficiency due to preheating to $1200 \mathrm{~K}$ is some $58 \%$ and almost $29 \%$ of the maximum permitted by the second law.

\section{Conclusions}

The above treatment is confined to theoretical limits for adiabatic conditions. Also, heat loss from system is neglected, specific heat capacity and mass flow rate remained constant and $Z T=1$ over the temperature range investigated. The results proffer novel designs which substantially improves the overall efficiency of the converter system which may well make small-scale thermoelectric power generation from regenerative combustion systems attractive in practice.

\section{Acknowledgment}

We thank Dr Paul Ronney for helpful comments.

\section{References}

[1] Weinberg F J 1971 Combustion temperatures: the future? Nature 233239

[2] Lloyd S A and Weinberg F J 1974 A burner for mixtures of very low heat content Nature 25147

[3] Lloyd S A and Weinberg F J 1975 Limits to energy release and utilisation from chemical fuels Nature $\mathbf{2 5 7} 367$

[4] Hardesty D R and Weinberg F J 1976 Converter efficiency in burner systems producing large excess enthalpies Combust. Sci. Technol. 12153

[5] Weinberg F J 1986 Combustion in heat-recirculating burners Advanced Combustion Methods (London and New York: Academic) chapter 3

[6] Rowe D M and Bhardari C M 1983 Definitions and basic principles ed Holt, Rinehart and Winston Modern Thermoelectrics chapter 3 